\title{
Array-enhanced synchronization and coherence resonance in coupled excitable semiconductor lasers
}

\author{
Auro M.Perego ${ }^{1, *}$ and Marco Lamperti ${ }^{2}$ \\ 1. Aston Institute of Photonic Technologies, Aston University, Birmingham, B4 7ET, UK \\ 2. Dipartimento di Fisica - Politecnico di Milano, Via Gaetano Previati 1/C, 23900 Lecco, Italy \\ "peregoa@aston.ac.uk
}

We present a numerical study of the nonlinear dynamics of a population of coupled semiconductor lasers with saturable absorber operating in the excitable regime [1] and described by a set of coupled Yamada models [2]. In particular we have investigated the self-organized synchronization process taking place spontaneously among the lasers, showing significant correlations between the spike-like pulses emitted by different lasers. Our findings demonstrate that synchronization in time and also in intensity occurs in a large region of the parameter space and for different population sizes and furthermore it is robust with respect to random distribution of the lasers' pump parameter which is linked to the excitability threshold.

Interestingly the width of the region in parameter space for which synchronization is stronger increases with the number of coupled elements, hence we are in presence of an array-enhanced synchronization.

The collective dynamics affects significantly the behaviour of the single laser, in particular reducing both the jitter and the intensity fluctuations in the pulse train of the single laser. We have found that the coherence resonance which usually takes place in the single excitable laser, corresponding to a minimum of the jitter as a function of the injected noise intensity, occurs in a broader region of the parameter space due to the mutual coupling among the lasers (array-enhanced coherence resonance). Our findings suggest interesting directions for further studies of excitable lasers both towards technological applications as well as in the fascinating field of optical emulation of neuromorphic systems[3, 4].

a)

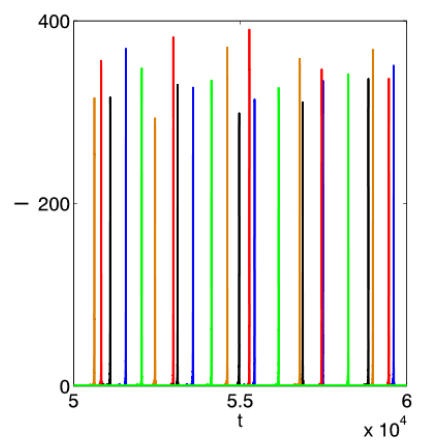

b)

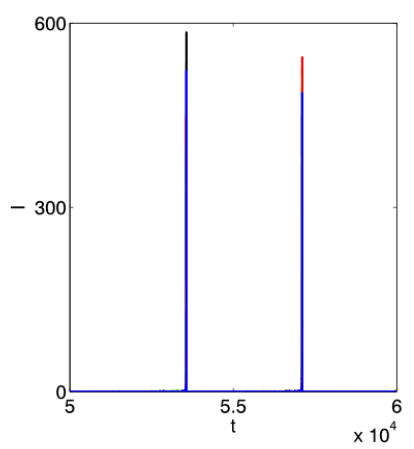

c)

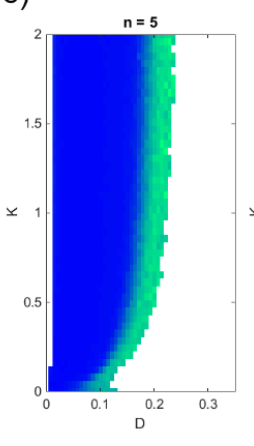

d)

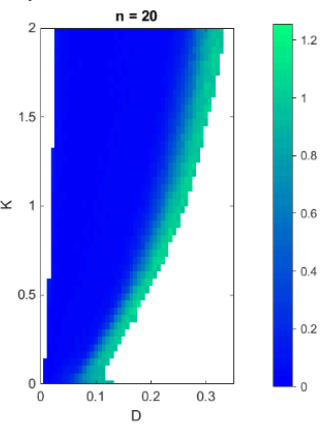

Fig. 1 Pulse train for five lasers in the uncoupled a) and coupled b) regime: synchronization is clearly visible in presence of coupling; different colours correspond to intensity (I) time (t) traces of different lasers; in the synchronized regime b), the pulses are overlapping in time. Array enhanced coherence resonance c), d): the single laser jitter is plotted versus noise strength $D$ and coupling $K$ : for higher number of coupled lasers d) the single laser jitter is low for a much larger interval of noise strength $D$ values at fixed coupling $K$; the letter $n$ on the plots denotes the number of coupled lasers considered.

\section{References}

[1] J. L. A. Dubbeldam, B. Krauskopf, and D. Lenstra, "Excitability and coherence resonance in lasers with saturable absorber," Phys. Rev. E 60, 6580 (1999).

[2] A. M. Perego and M. Lamperti, "Collective excitability, synchronization and array-enhanced coherence resonance in a population of lasers with a saturable absorber," Phys. Rev. A 94, 033839 (2016).

[3]P. R. Prucnal, B. J. Shastri, T. Ferreira de Lima, M. A. Nahmias and A. N. Tait, "Recent progress in semiconductor excitable lasers for photonic spike processing", Advances in Optics and Photonics 8, 228 (2016).

[4] B. Romeira, R. Avó, José M. L. Figueiredo, S. Barland and J. Javaloyes, "Regenerative memory in time-delayed neuromorphic photonic resonators" Scientific Reports 6, 19510 (2016). 